\title{
Advantages and Limitations of Magnetic Source Imaging
}

\author{
Samuel J. Williamson, Zhong-Lin Lü, Daniel Karron, and Lloyd Kaufman
}

\begin{abstract}
Summary: The term "magnetic source image" (MSI) describes the distribution of neuronal activity in the brain that can be deduced from measurements of the field pattern it produces across the scalp. The signals which provide the basis for an MSI are obtained from the magnetoencephalogram (MEG) which is conventionally recorded with superconducting detectors. Advances in MSI techniques during the past decade have revealed numerous aspects of the functional organization of human sensory systems that were previously unknown. In addition, studies of spontaneous signals, such as those in the alpha bandwidth, have identified specific cortical areas that support rhythmic activity. Extensions of this approach to cognitive research are able to determine the active cortical areas where spontaneous activity is suppressed when a person is engaged in a task such as mental imagery and auditory memory recall. Because only the component of the intracellular current tangential to the overlying skull contributes to the extracranial field, a confined source - modeled as a current dipole - has a characteristic field pattern that simplifies the pattern recognition problem of identifying the underlying sources. This advantage is illustrated by the identification of simultaneously active sources in auditory primary and association cortex. Their separate localization makes it possible to characterize their functional differences. Because the source strength in an MSI may be inferred without knowledge of the electrical conductivities of intervening tissue, it is also possible to estimate the extent of cortical envolvement. From the tangential source strength in an MSI, it is possible in most cases to determine the total source strength by taking account of the orientation of the cortical surface. This provides an objective, quantitative measure of the strength of neuronal activity. At present, the major limitation in more extensive use of MSI is the cost of instrumentation. While it requires no contact with the head, and measurements can commence within a few minutes of the arrival of the subject or patient, the present cost of a large array of sensors is two to three million dollars.
\end{abstract}

Key words: MSI (magnetic source image); MRI (magnetic resonance image); Current dipole; Neuronal activation; Habituation; Spontaneous cortical activity.

\section{Introduction}

Measurements of voltages across the scalp (EEG) and magnetic fields near the scalp (MEG) provide complementary measures of the underlying neuronal activity. The most pronounced difference between the two approaches in our view does not come from the information that is available in the signals but rather from the different emphasis traditionally placed on how data are analyzed. On the whole, EEG studies emphasize the pattern of electric potential across the scalp, whereas MEG studies emphasize the underlying neuronal ac-

Neuromagnetism Laboratory, Departments of Physics and Psychology and Center for Neural Science, New York University, New York, NY, U.S.A.

Accepted for publication: September 16, 1991.

This research was supported by grant AFOSR-90-0221 from the Air Force Office of Scientific Research and by equipment support from Silicon Graphics, Incorporated. We thank Dr. N. Chase for access to the MRI facilities at the NYU Medical Center and J. Stephenson for helping with MR recordings.

Correspondence and reprint requests should be addressed to Prof. Samuel J. Williamson, Department of Physics, 4 Washington Place, New York University, New York, NY 10003 U.S.A.

Copyright (C 1991 Human Sciences Press, Inc. tivity. Indeed, the term "magnetic source image" (MSI) has come to be associated with magnetic analysis. The word "emphasize" is used advisedly, since there are many notable exceptions to this generalization. In part the difference in emphasis comes from the EEG having been exploited earlier, and because of that there are more widespread clinical applications. This fosters a more phenomenological approach for purposes of facilitating classification schemes to identify abnormal functions.

Locating a neuronal source magnetically is easier than electrically, because to good approximation a simpler model can be used for the head. A sphere will suffice, in many cases, fit to the curvature of the inner surface of the skull in the region over the source (Hari and Ilmoniemi 1986). The conductivity of intervening tissue has no effect on the field pattern (Grynszpan and Geselowitz 1973), and thus need not be taken into account when deducing the location and strength of the source (Williamson and Kaufman 1987). By contrast, the values of conductivity enter directly in determining the pattern of electric potential across the scalp. Lack of these data for individual subjects may well be one reason why the EEG literature is practically devoid of values specifying neuronal source strengths, for instance as given by the 


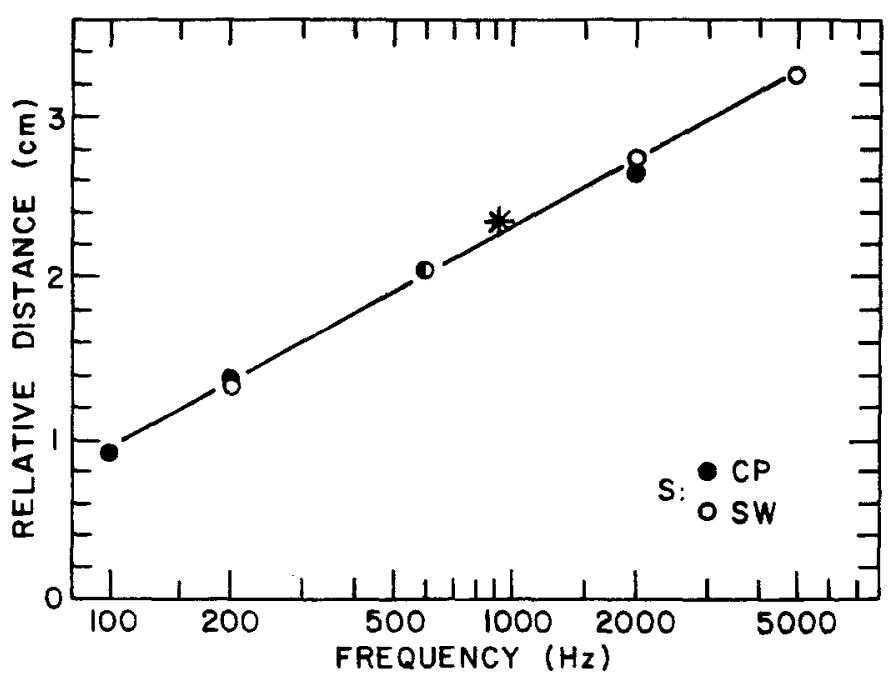

Figure 1. Cumulative distance in 3-D space from the site of one response to the next for steady-state stimuli at the indicated tone frequencies. Only the relative slopes should be compared, because the origins of the best-fitting lines were shifted to a common value of zero for 20 $\mathrm{Hz}$. Stimuli were tones with amplitudes modulated at 33 $\mathrm{Hz}$, and the field patterns for the $33 \mathrm{~Hz}$ responses were mapped to obtain the locations for individual sources. All points lie within $1 \mathrm{~mm}$ of the best line. The star indicates the relative location of the response to amplitude-modulated noise whose power spectrum peaks at $900 \mathrm{~Hz}$ (From Romani et al. 1982b).

current dipole moment of a patch of active cerebral cortex. On the other hand, moments are often quoted in the MSI literature. The value of the moment provides an objective measure of the strength of neuronal activity. In addition, for long-latency components of the event related field (ERF), it can be interpreted to provide an estimate for the spatial extent of the corresponding cortical activity (Lü and Williamson 1991).

For these reasons, there is growing interest in exploiting MSIs to obtain information about the functional organization of the brain. Systematic measurements have been carried out where a current dipole source is placed at a known location inside a spherical or realistic representation of the skull, with a homogeneous conducting medium inside. These have achieved a consistent accuracy of better than $3 \mathrm{~mm}$ in locating the dipole when repeated sets of measurements are compared (Yamamoto et al. 1988). Such accuracy can be achieved only if the field pattern is properly sampled, with measurements taken on all sides of areas where the inward or outward directed field is strongest (the "field extrema"). Also, it is important to determine the 3-D position and orientation of the sensor, to take account of the direction of the measured field component when fitting a source model to the data. Failure to carry out both of these procedures is likely to yield much poorer results (Cohen et al. 1990).

\section{Locating Sources Accurately}

The first neuromagnetic study to locate a neuronal source was carried out by Brenner et al. (1978) on the cortical response to stimulation of a finger. The procedure subsequently revealed that response components with latencies as long as 150 msec originate in somatosensory cortex (Kaufman et al. 1981), a feature which had not previously been established in EEG studies. Moreover, the field pattern across the scalp in response to stimulation of various fingers revealed aspects of the somatosensory humunculus (Okada et al. 1984). Detailed measurements also established separate somatosensory and motor contributions in a task involving voluntary movement of a finger (Okada et al. 1982). Similar neuromagnetic techniques applied to auditory evoked responses have identified a feature not previously known about the functional organization of human cortex: the tonotopic organization of the primary auditory cortex (Romani et al. 1982; Pantev et al. 1988). Indeed, the precision - as distinguished from accuracy - for determining the relative positions of the cortical responses may be estimated from the scatter of deduced locations about the best-fitting line that describes the tonotopic progression. All of the locations for two subjects lie within $1 \mathrm{~mm}$ of a logarithmic function for the tonotopic sequence for the steady-state response (Romani et al. $1982 \mathrm{~b}$ ), as illustrated in figure 1. High accuracy has also been obtained for the tonotopic organization subsequently observed for the $\mathrm{N} 100 \mathrm{~m}$ transient response (Hoke 1988) (the ' $m$ ' identifies this component as being observed magnetically). In sum, the central motivation for MSI research has been the technique's ability to elucidate brain functions by localizing neuronal activity with high precision and relatively high accuracy (Hari and Lounasmaa 1989).

In less rigorous conditions, the reproducibility in determining the locations of the $P 50 \mathrm{~m}, \mathrm{~N} 100 \mathrm{~m}$, and P200m transient components of the auditory evoked response to a tone burst has been assessed for individual subjects. Positions over the right hemisphere were reproduced to within $3-10 \mathrm{~mm}$ acróss sessions on different days in each of 12 subjects (Baumann et al. 1990). From a wide variety of results obtained with careful studies, we conclude that with a favorable geometry (sources at positions where the skull is well-modeled by a sphere), reasonably strong and confined neuronal activity, and intelligent care, it is possible to achieve an accuracy of $3 \mathrm{~mm}$ in locating that activity. More general- 


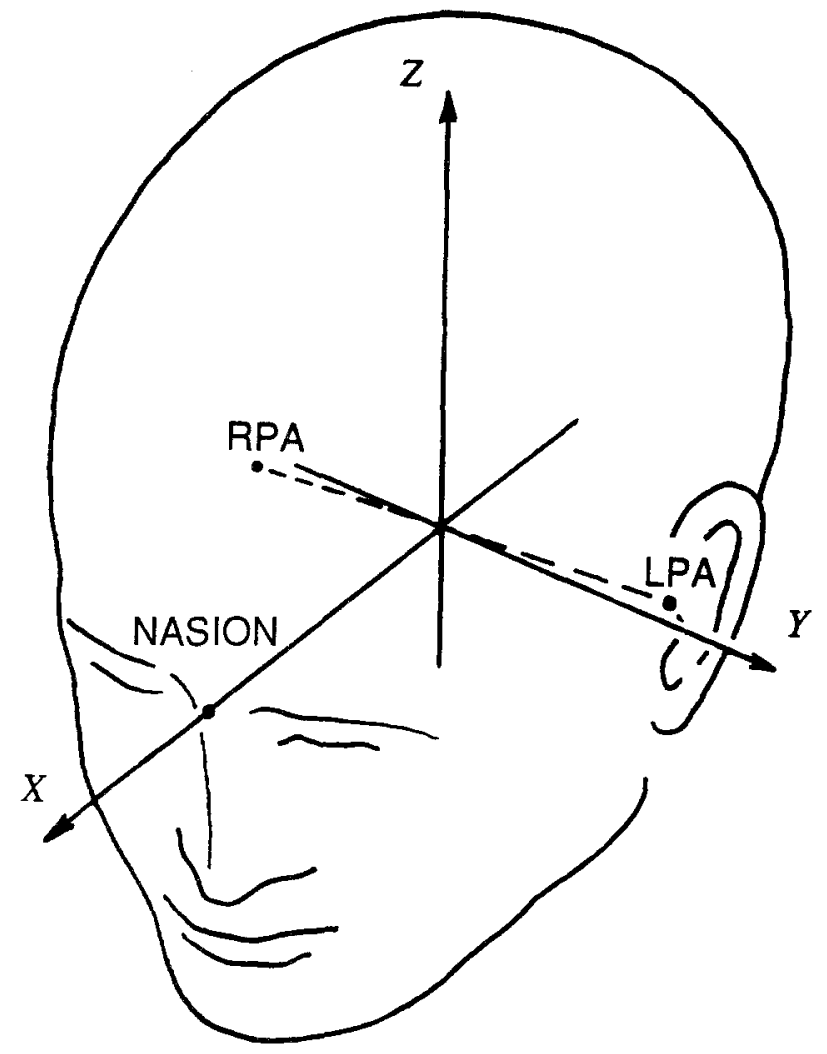

Figure 2. PPN head-based coordinate system for specifying positions within the head or near the scalp of a subject.

ly, it would be reasonable in somewhat less favorable cases to expect an accuracy of better than $1 \mathrm{~cm}$ for activity in cortical sensory areas.

\section{Relating Structure and Function}

Determining the locations of neuronal activity in space is of little value unless the anatomical substrate can be identified accurately. It is increasingly common to relate deduced positions to features of a magnetic resonance image (MRI) for this purpose. The challenge then is to establish a common coordinate system for specifying MRI pixels and MSI sources. We have chosen the three reference points of the 10-20 system as the "cardinal landmarks" for defining an appropriate system (Williamson and Kaufman 1989). These are the left and right periauricular points and nasion. For convenience, we call this coordinate system the "PPN head-based system". As a short-hand, we refer to coordinates in this system as "PPN coordinates". The coordinate axes of the PPN system are defined in the following way (figure 2). The origin is the mid-point between left and right periauricular points. The $x$-axis passes through the origin to emerge from the head at the nasion. The $z$-axis is oriented perpendicular to both the $x$-axis and the line between periauricular points, and passes through the origin to emerge near the vertex. It need not pass through the vertex, and if one ear is lower on the head than the other it generally will not lie parallel to the longitudinal fissure. The $y$-axis is perpendicular to both the $x$ - and $z$-axes and passes through the origin to emerge from the left hemisphere. Generally it will pass near, but not through, the periauricular landmarks. The PPN System defined this way is a right-handed coordinate system.

To specify measurement positions, as well as the corresponding sensor orientations, this coordinate system can be established for a given subject by using an appropriate 3-D digitizer, such as the Polhemus 3-Space (Polhemus Navigation Corporation), to characterize the relative positions of cardinal landmarks. During measurements, the same system can be employed to register field measurement positions and sensor orientations with respect to the PPN System (Yamamoto et al. 1988). Alternatively, this can be achieved by using the sensors themselves to locate reference coils placed at known locations on the skin (Erné et al. 1987).

Similarly, MRI recordings must contain accurate information about the cardinal landmarks. One traditional method is to place a marker, such as a vitamin-E pill, on the skin at each cardinal landmark for recordings. We have developed a method that achieves registration with higher accuracy, and we believe is easier to use and more convenient (Karron et al. 1991). Three "pointers" are held by a Velcro head-band worn by the subject so that each is directed toward a cardinal landmark, as illustrated in figure 3. The pointer itself consists of 4 thin tubes (called "spokes") filled with vitamin $\mathrm{E}$ that are machined so that they cant inward toward a common point in space. That point coincides with the resting spot when the pointer is placed against the skin at one of the cardinal landmarks.

When an MRI is recorded, several additional slices must be registered outside the head to cover the regions of the pointers. An MR slice that intersects the spokes registers one spot for each of them, as shown in figure 4. When the MRI is displayed on a computer monitor, the operator moves a cursor to each spot in turn and a program computes the MRI coordinates for the exact center of the spot. The location is therefore determined with greater accuracy (better than $0.3 \mathrm{~mm}$ ) than the size of an image pixel $(0.8 \mathrm{~mm})$. This procedure of pointing to each spot is repeated for each MR slice that shows them. Then the programed computer computes the bestfitting line for each set of spots belonging to a given spoke, and it determines where the spokes intercept. More specifically, it computes the point in space that lies closest to all the spokes of a given pointer. We find the location where the pointer touches the skin is thereby 


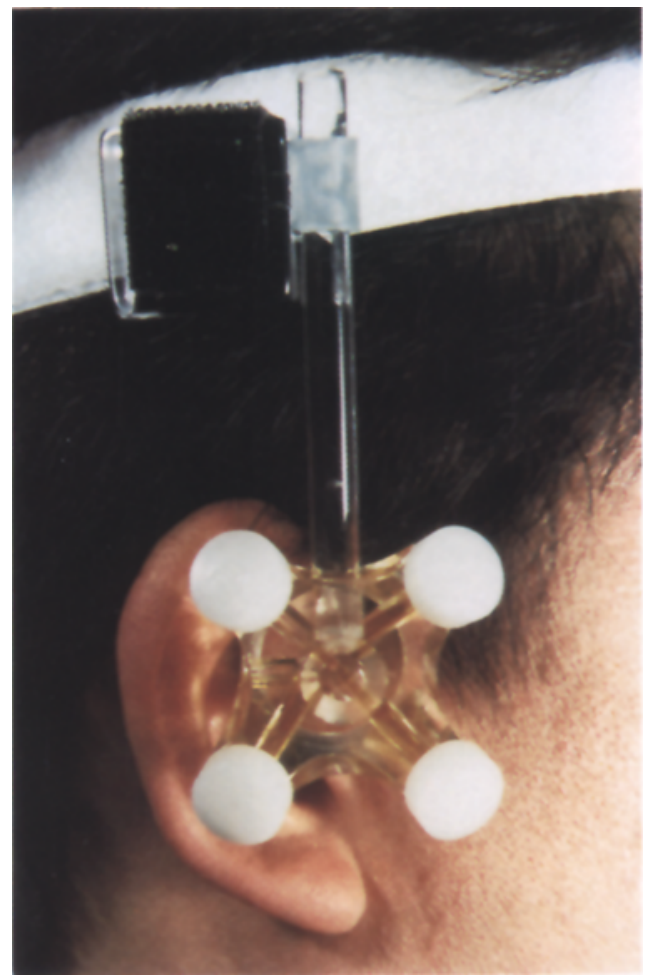

Figure 3. A plexiglas pointer is held against the right periauricular landmark of a subject for an MRI scan. The arrangement of the supporting rod where it is held at the upper end to a plexiglas cube permits adjustments of position and angle. The cube has a piece of Velcro glued to its base so that it can be attached to the subject's Velcro headband in any direction. The yellow spokes of the pointer can be seen directed inward to the periauricular landmark on the scalp.

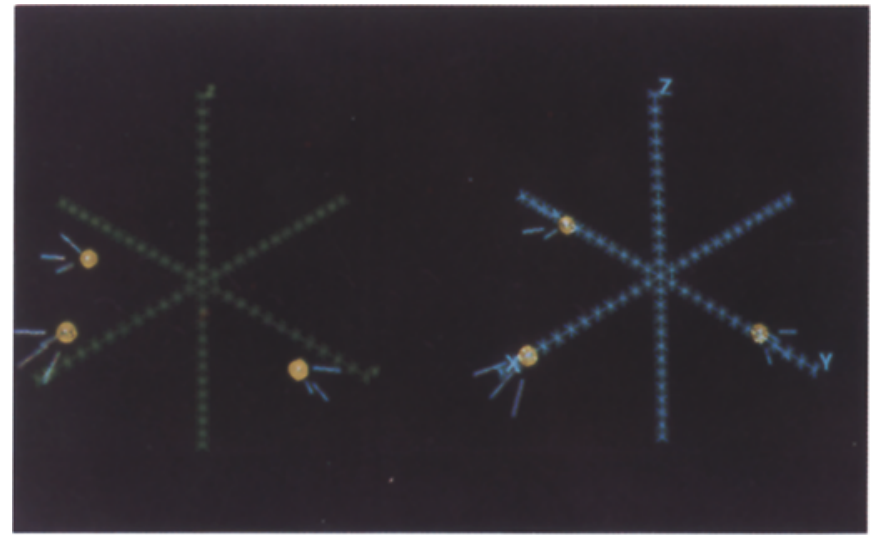

Figure 5. Computer representation of three pointers showing the spokes directed toward cardinal landmarks (yellow). The green axes define the cartesian coordinate system in which MRI pixels are specified. The blue axes define the PPN head-based system, which is defined with respect to the cardinal landmarks.

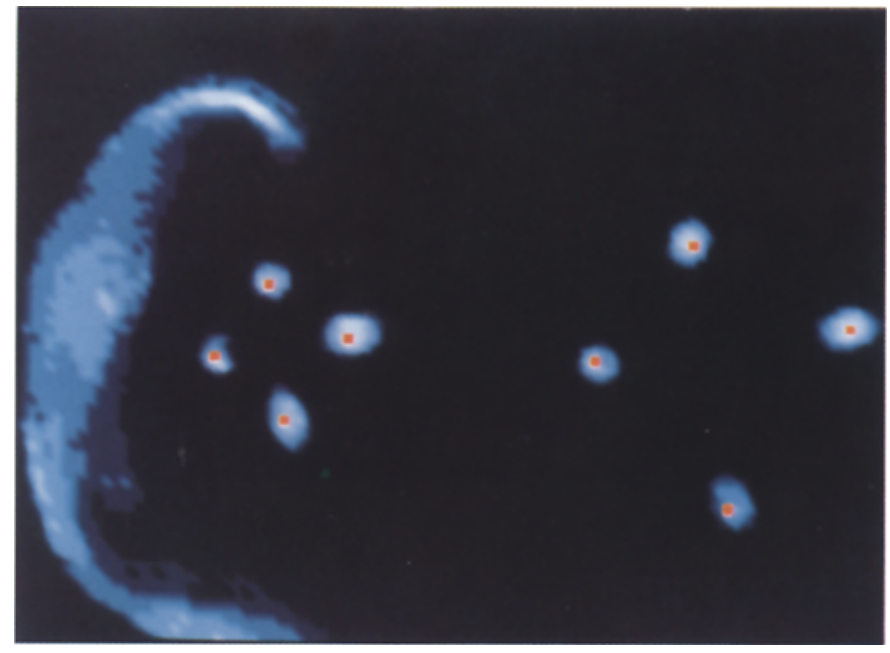

Figure 4. Sagittal MRI scans of a pointer held against the right periauricular point of a subject. (Left panel) Scan closest to the scalp, showing the ear, four spots depicting the four spokes of the pointer, and red dots indicating the pixels closest to the center of each spot. (Right panel) Scan closest to the outer ends of the four spokes. These images were photographed from a Sillicon Graphics $85 \mathrm{GT}$ computer monitor.

established with an accuracy of better than $0.4 \mathrm{~mm}$. In practice, the accuracy of determining each cardinal landmark is therefore limited by how accurately the pointer can be positioned on the skin for the MRI recording.

This analysis establishes the positions of the three cardinal landmarks with respect to the MR coordinate system. Therefore, the location of each pixel of the MR image can be expressed in the same PPN system as the neuromagnetic measurements (figure 5). By this means, neuronal function can be related to anatomical structure.

\section{Spherical Model for the Head}

To interpret the field pattern measured across the scalp, the actual shape of the head must be taken into account. The spherical model has provided a convenient simplification for modeling the pertinent region of the head when interpreting neuromagnetic data (Grynszpan and Geselowitz 1973; Cuffin and Cohen 1977). Hari and Ilmoniemi (1986) provide evidence through a model calculation that the sphere is best when fit to the region of the head overlying the source, where the volume currents are strongest. Because the curvature of the skull differs from one location to another, the spherical model will have a different size than the subject's head. This is illustrated in figure 6 for a fit to the region over the parieto-occipital sulcus. A computer cursor was employed to record the representative locations on the inner surface of the skull on appropriate MR slices, and 


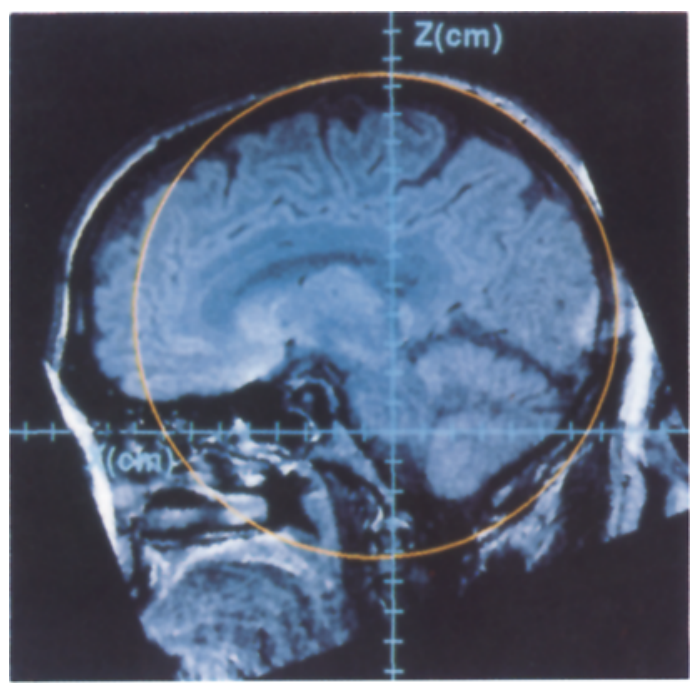

Figure 6. Spherical model (orange) best fitting the parietal region of the inner surface of the skull, shown in relation to a sagittal MRl cross section of the head. PPN axes are depicted in blue.

the computer subsequently computed the location of the center of the sphere whose surface most closely agrees with the locus of points.

Several groups are presently developing realistic conductivity models for the head that can be used with finite element methods to compute the extracranial field and scalp potential for a model source within. Hämäläinen and Sarvas (1989) used a numerical approach to determine the importance of including the currents within the skull and dermis when deducing an MSI within a realistically shaped head. The medium within the skull was assumed uniform in conductivity, and the skull itself was assumed of uniform thickness. They concluded that contributions to the field from the weak currents in the skull and dermis can be neglected for many purposes. Moreover, current patterns produced by departure from sphericity of the inner surface of the skull contribute fields that are generally weak except for sources that are deeper than $4 \mathrm{~cm}$. Considerable improvement in source localization can be achieved by employing numerical models to account for actual volume current patterns (Rose et al. 1989). The effect of using spheres determined by different criteria to model the head when locating a source in the temporal area has been assessed by Lütkenhöner et al. (1990). The most important contributions from non-sphericity are in frontal areas, and they can be appreciable for sources lying more than $2 \mathrm{~cm}$ below the skull. Nevertheless, localization accuracies of better than $1 \mathrm{~cm}$ can be achieved with numerical modeling to take into account the actual shape of the skull.

\section{Multiple Neuronal Sources in Temporal Cortex}

As the quality of MEG measurements improves, interest can be extended to situations where several closelying sources contribute to the field pattern at any given moment. Weinberg et al. have discussed one example in which several dipoles were placed within a model skull and were activated in various combinations (Weinberg et al. 1986). An ambiguity arises when it is not clear which field extrema are associated with a given source. One method for dealing with the multiple dipole case has been developed by Scherg (1990) for EEG data, when sources are activated in succession. We shall illustrate another method that has proved effective where the individual sources can be separated by exploiting their functional differences.

We shall illustrate the resolution provided by MSI with a difficult challenge that had not previously been met with EEG techniques: resolving the sources in temporal lobe that contribute to the 100-ms component of the auditory evoked response. Näätänen and Picton (1987) suggest that as many as 6 different neuronal sources may contribute in some measure to the event related potential (ERP) detected $100 \mathrm{~ms}$ following onset of a tone burst. Evidence for a source of $\mathrm{N} 100$ in the supratemporal plane was obtained by Vaughan and Ritter (1970), who observed a polarity reversal in the EEG over that location when the nose was used as reference. Definitive identification of the actual locations was provided by neuromagnetic studies by Hari et al. (1980) and Bak et al. (1981). Contributions from an additional source in the temporal lobe were inferred by Wolpaw and Penry (1975) who reported statistically significant differences in the EEG waveform within the interval of 100 to $200-\mathrm{ms}$ from an electrode over the vertex compared with another over temporal scalp positions $\mathrm{T} 3$ and T4. The difference was called a "T" complex, consisting of a positive peak at about $105-110 \mathrm{msec}$ and negative peak at $150-160 \mathrm{msec}$. More recent studies of scalp potentials also have been interpreted as indicating the presence of a lateral temporal source (Vaughan et al. 1980; Wood 1982). The fact that several neuronal sources contribute differently to the ERP and ERF measured near the scalp was made clear by Hari et al. (1982) who found that the amplitudes of the 100 msec electric and magnetic responses did not increase in the same way with increasing interstimulus interval (ISI). The two would be proportional if they originated form the same source of fixed geometry.

In studies of neuromagnetic responses to tone bursts applied with a constant interstimulus interval (ISI), Lü et al. (submitted) observed that when the ISI is lengthened a second source can be detected with a latency $10 \mathrm{~ms}$ shorter than that of the $\mathrm{N} 100 \mathrm{~m}$ component. In other 

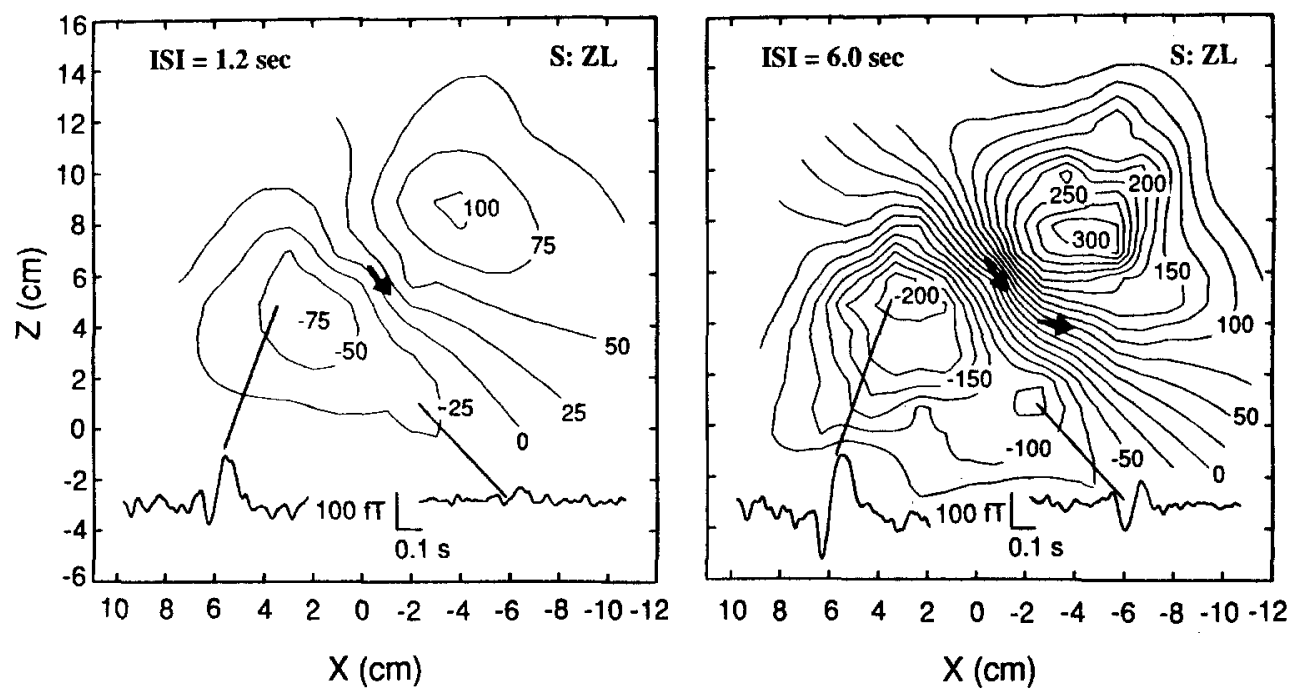

Figure 7. Sagittal view depicting the isofield contours over the left hemisphere for a subject responding to tone bursts presented at a fixed ISI of $1.2 \mathrm{sec}$ and $6 \mathrm{sec}$.

words, the field map over the temporal and parietal areas indicates the presence of the classic N100m response for an ISI of $1.2 \mathrm{sec}$ (figure 7a), but for an ISI of $6 \mathrm{sec}$ the pattern reveals the presence of a second source as well (figure $7 \mathrm{~b}$ ). For convenience, we shall refer to this new component as the latent component and denote it by $\mathrm{L} 100 \mathrm{~m}$. This terminology comes from the dictionary definition of latent as "not apparent but capable of being expressed".

The striking differences between the two patterns can be seen in the appearance for long ISI of a region of strong negative field over the area of the ear, and a strengthening and shift to the lower right of the upper right positive extremum, so that it is much stonger than any portion of the negative extrema at the left. A reasonable hypothesis is that neuronal activity appears which produces a positive field extremum at the upper right nearly coinciding with the existing positive extremum of $\mathrm{N} 100 \mathrm{~m}$. Its negative extremum lies over the region of the ear, to the lower right of the negative extremum of $\mathrm{N} 100 \mathrm{~m}$.

A local sphere model was employed to determine the locations of the two neuronal sources. Its center was determined by the curvature of the inner portion of the skull, extending over a radius of $5 \mathrm{~cm}$ about the point lying about midway between the estimated source positions. Table 1 lists the parameters of the dipole that best account for the N100m pattern at short ISI, for each of 2 subjects. When a second dipole is added to account for the pattern at long ISI, the position and orientation of this $\mathrm{N} 100 \mathrm{~m}$ dipole were first fixed and then a 6-parameter fit was employed to determine its new strength and the 5 parameters of the $\mathrm{L} 100 \mathrm{~m}$ dipole. The result for sources in the left hemisphere of one subject is shown in table 1. In a second fitting procedure, we used these dipole parameters as the best initial estimate and carried out a 2-dipole fit, allowing all 10 parameters to vary. The best-fitting positions for the first and second procedures varied by less than $4 \mathrm{~mm}$.

\section{Ambiguities in Identifying Sources}

As mentioned earlier, there is an inherent ambiguity in assigning field extrema to individual sources in a 2-dipole model. A positive extremum can be associated

Table 1. Best-fitting parameters for the dipoles that best account for the field pattern at both short and long ISI for the subject characterized in figure 7b. Parameters at short ISI condition for the subject as characterized in figure 7a and for another subject are also shown.

\begin{tabular}{||c|c|c|c|c|c|c|c|}
\hline \hline Condition & Subject & Component & $x(\mathrm{~cm})$ & $y(\mathrm{~cm})$ & $z(\mathrm{~cm})$ & $\Psi$ (degree) & $Q(n A \cdot m)$ \\
\hline \multirow{2}{*}{ Short ISI } & ZL (Left) & $N 100 m$ & -0.9 & 6.3 & 6.2 & -140 & 3.3 \\
\cline { 2 - 8 } & SW (Left) & $N 100 m$ & 0.8 & 4.3 & 5.4 & -134 & 11.4 \\
\hline \multirow{2}{*}{ Long ISI } & \multirow{2}{*}{ SW (Left) } & $N 100 m$ & 1.2 & 5.2 & 5.9 & -122 & 11.4 \\
\cline { 3 - 8 } & & L100m & -1.9 & 6.7 & 4.2 & -94 & 2.3 \\
\hline
\end{tabular}


Table 2. Successive sets of parameters describing two dipoles in the left hemisphere that are being adjusted to achieve a best fit to the isofield contours of figure $7 \mathrm{~b}$ for $\mid \mathrm{SI}=6 \mathrm{sec}$. Orientations of the two dipoles are illustrated schematically at the right by arrows. Between steps 24 and 25 the orientations of the dipoles are switched as are two of the three position coordinates. However, they are switched back to the original assignments between steps 26 and 27. Each step comprises 6 computer iterations.

\begin{tabular}{|c|c|c|c|c|c|c|c|c|}
\hline Step No. & Dipole & $Q x(n A \cdot m)$ & $Q z(n A \cdot m)$ & $X(m m)$ & $Y(\mathrm{~mm})$ & $Z(\mathrm{~mm})$ & Dipole 1 & Dipole 2 \\
\hline \multirow{2}{*}{24} & 1 & -2.5 & -8.2 & -4.8 & -72.3 & 58.4 & & \\
\hline & 2 & -10.0 & -2.3 & 10.2 & -56.0 & 45.8 & & \\
\hline \multirow{2}{*}{25} & 1 & -2.7 & -2.2 & -5.5 & -77.0 & 62.7 & & \\
\hline & 2 & -2.7 & -10.8 & -4.1 & -70.4 & 41.7 & & \\
\hline \multirow{2}{*}{26} & 1 & -3.6 & -5.0 & -3.7 & -73.8 & 61.5 & & \\
\hline & 2 & -3.9 & -7.6 & 1.3 & -65.0 & 40.1 & & \\
\hline \multirow{2}{*}{27} & 1 & -3.3 & -6.8 & -2.6 & .71 .9 & 60.6 & & \\
\hline & 2 & -6.9 & -5.4 & 3.7 & -60.0 & 40.9 & & \\
\hline
\end{tabular}

a priori with either of the negative extrema. To investigate how the program dealt with this, we followed the evolution of the fitting sequence and found instances when an attempt to switch associations was made. Table 2 provides one such example. However, for a variety of starting conditions in which the $\mathrm{N} 100 \mathrm{~m}$ dipole is close to that providing the best fit to the data of figure $7 \mathrm{a}$ the minimization procedure based on chi-square as the cost function yielded similar final values for the parameters. This demonstrates that the fitting procedure is robust.

Figure 8 shows the locations and orientations of the

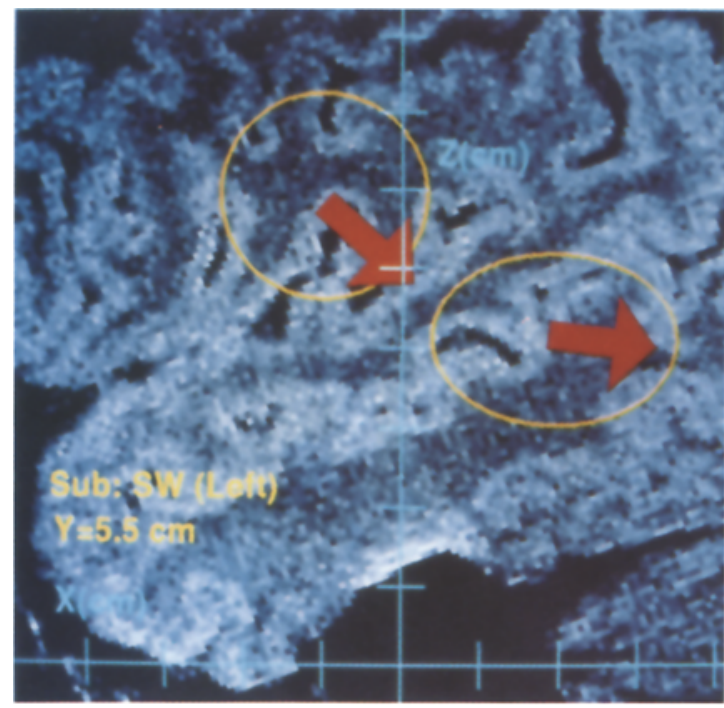

deduced N100m and $\mathrm{L} 100 \mathrm{~m}$ dipoles in the left hemisphere with respect to a sagittal MRI recorded near the average depth of the $1100 \mathrm{~m}$ and $\mathrm{N} 100 \mathrm{~m}$ sources. The N100m source lies $5 \mathrm{~mm}$ from the sulcus depicted in this superficial MRI slice. At a greater depth beneath the scalp (e.g., at $y=-5 \mathrm{~cm}$ ) the sulcus comes closer to the position of the dipole. Moreover the dipole is oriented perpendicular to the average direction of the nearby sulcus. This can be understood if the neuromagnetic field is produced by intracellular currents within pyramidal cells of the cortical layer forming the floor of

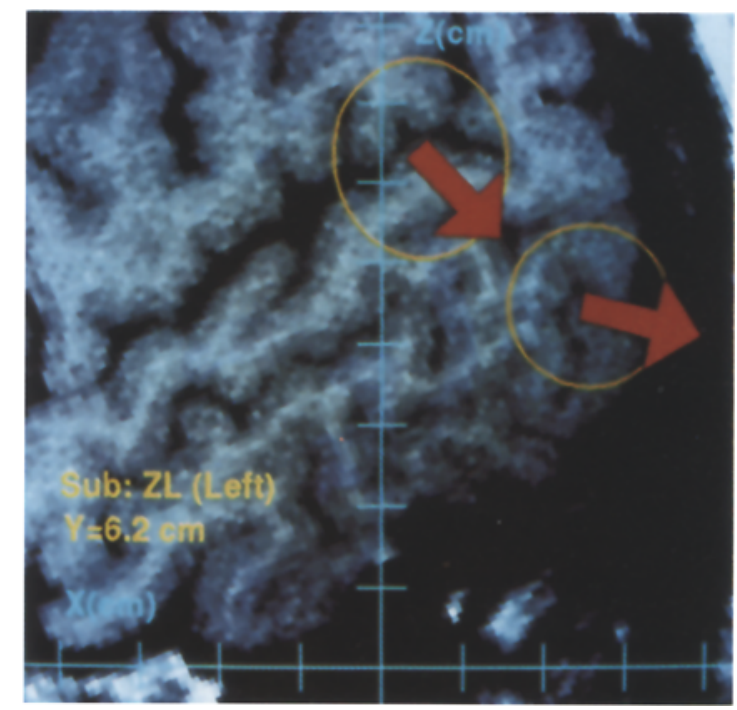

Figure 8. Locations and orientations of dipoles in the left hemisphere accounting for the field patterns in figure 7 (left panel; subject SW), as well for the field pattern of another subject (right panel; subject ZL) are shown as red arrows, where the tip of the stem of each arrow is placed at the respective source. Orange ovals depict the $95 \%$ confidence region for each location. Tic marks along the PPN $x$ and $z$ axes are spaced at $1 \mathrm{~cm}$ intervals. 


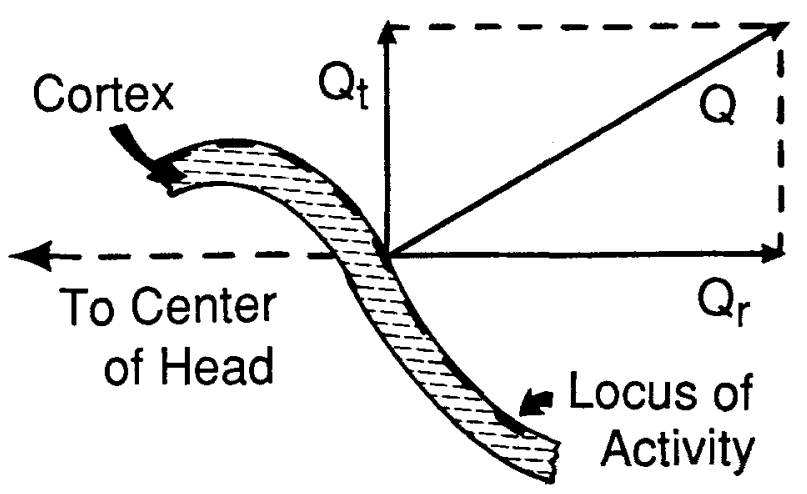

Figure 9. Projection of the tangential component $Q$ t of a current dipole onto the line that is perpendicular to the cortical surface to predict the total current dipole moment $Q$.

the lateral sulcus, in primary auditory cortex (Hari et al. 1980; Bak et al. 1981). By contrast the L100m source is found to lie within the supratemporal sulcus, in the region of the auditory association cortex (Pandya and Seltzer 1982), and it is oriented nearly perpendicular to the sulcus at its position.

Lü et al. (submitted) have also observed a second component with approximately $200 \mathrm{~ms}$ latency, for tone burst stimuli presented at a long ISI, whose source is located in this same region of the lateral temporal area, but this will not be discussed here.

\section{Total Source Strength}

When the pertinent region of the head is well-represented by a sphere, only the component of a current dipole source that is tangential to the sphere contributes to the external field (Grynszpan and Geselowitz 1973). In this sense, what neuromagnetic measurements will not "see" is a precisely radial source. This class of sources is a small proportion of the total. Some two-thirds of cerebral cortex is located within fissures and sulci, where the perpendicular to the cortical surface has an appreciable component lying tangential to the overlying scalp. Thus the majority of the cerebral cortex, as well as subcortical regions, will be accessible for magnetic study.

While only the tangential component $Q_{t}$ of a dipole will be directly recorded magnetically, it is nevertheless possible to infer the total current dipole moment $Q$ from knowledge of its tangential component $Q_{t}$. Anatomical information provides the necessary connection. In cerebral cortex, pyramidal cells are the dominant neuronal populations having a preferred orientation for the axes of their dendritic trees. The corresponding intracellular currents have a net direction perpendicular to the cortical surface. Therefore, it is only necessary to define the local normal to the cortical surface to infer the direction of the total dipole moment. Figure 9 illustrates how the observed tangential component $Q_{t}$ is projected onto the direction normal to the surface to predict the total dipole moment $Q$. This kind of analysis has been applied to obtain the total current dipole moments for steady-state responses to amplitude modulated tone of various frequencies (Romani et al. 1982). While the tangential component $Q_{t}$ varies considerably with tone frequency, the total moment $Q$ is nearly independent of frequency, suggesting that the same number of neurons respond regardless of frequency.

Once the total dipole moment is obtained, it is possible to take a further step and deduce the areal extent of cortex that responds to the stimulus. An analysis of current source-density measurements for long-latency responses in cat and monkey has shown that the current dipole moment at the moment of peak activity is about $50 \mathrm{pA} \cdot \mathrm{m}$, to within an uncertainty of about a factor of 2 , for each $\mathrm{mm}^{2}$ of cortical surface area (Lü and Williamson 1991). Therefore, the total active cortical area can be estimated as $Q /\left(50 \times 10^{-12}\right) \mathrm{mm}^{2}$.

\section{Spontaneous Rhythms of Cerebral Cortex}

Because neuromagnetic fields are comparatively well confined near their sources, it is possible to investigate aspects of spontaneous cortical activity that have not yet been studied with EEG measurements. A series of cognitive studies we have carried out is motivated by the research of Pfurtscheller et al. (1977, 1988, 1988b) who found that EEG power in the alpha band between 8 and $13 \mathrm{~Hz}$ suffers a sharp reduction ("event related desynchronization") subsequent to visual stimulation as well as during the performence of voluntary acts. Their method is similar to one devised by Kaufman and Price (1967) to study modulation of high-frequency EEG by visual stimulation, and by Kaufman and Locker (1970) to study effects of attention on visual modulation of alpha activity.

We have carried out similar studies with MEG measurements over various areas of the scalp and find that magnetic alpha rhythm during cognitive tasks is sharply suppressed over the areas of cerebral cortex that may be expected to participate. For instance, field maps over the occipital and parietal areas provide evidence that visual cortex plays a role in visual imagery (Kaufman et al. 1990). The strongest source of alpha rhythm is the parieto-occipital sulcus, where sources ("alphons") of individual alpha spindles have been localized (Williamson and Kaufman 1989b). Nevertheless, there is a greater percentage of power suppression near the midline of the occipital area during mental imagery, which is the key 


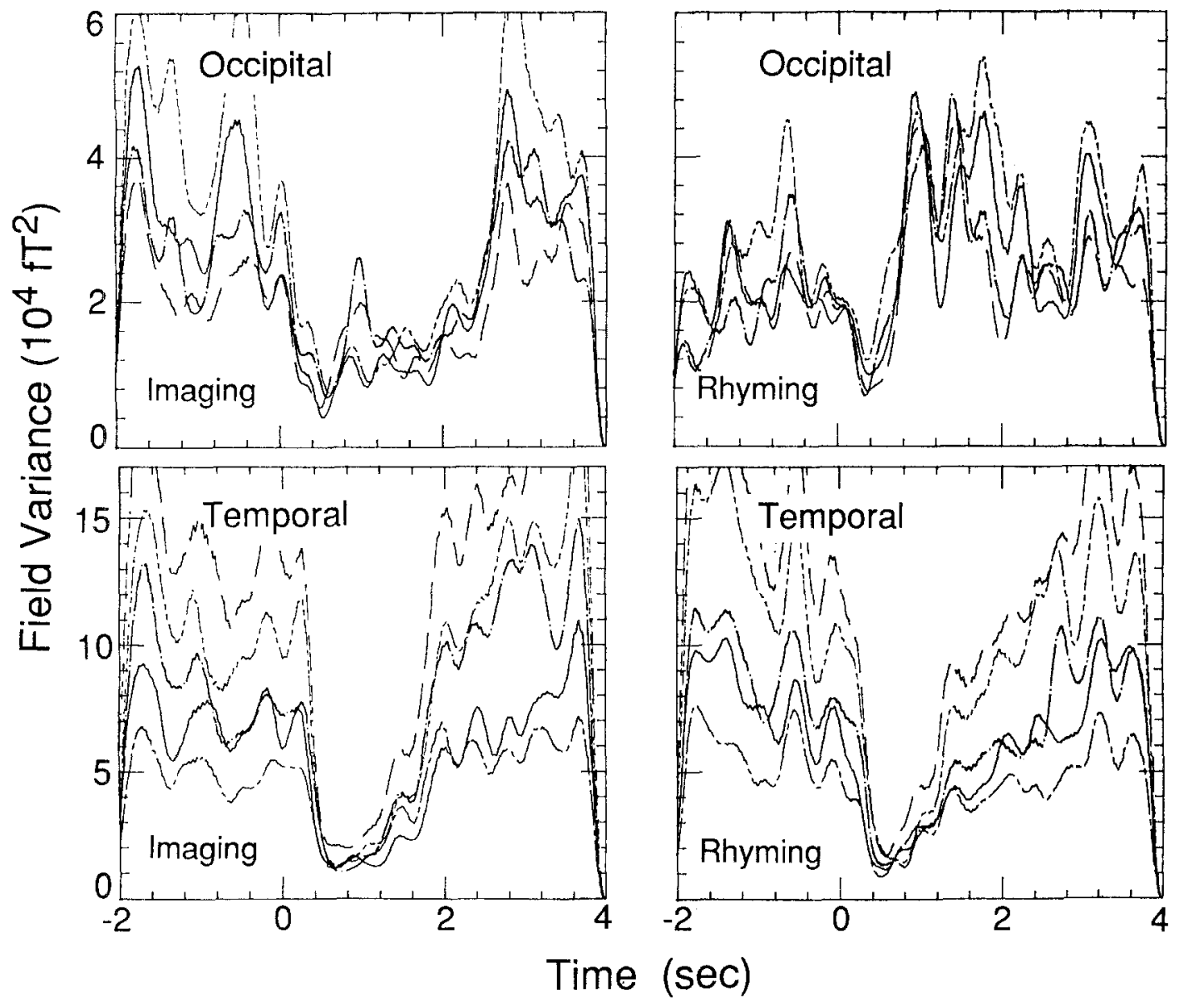

Figure 10. Variance in field power within the alpha bandwidth averaged over 36 trials when an imageable or nonimageable word is shown on a display for $200 \mathrm{msec}$ beginning at the time origin. Data were low-pass filtered to smooth over individual alpha oscillations. Five traces are provided by simultaneous measurements with a probe consisting of four sensors evenly spaced on a circle of 2 -cm radius centered on the fifth sensor. The probe was positioned over the occipital scalp (upper panels) or anterior temporal area (lower panels). The left panels show suppression when the subject seeks an image for the object that an imageable word represents, with suppression beginning earlier and enduring longer over the occipital area than temporal. The right panels show the measurements at the same respective locations when the subject seeks a word that rhymes with a non-imageable word displayed. Occipital suppression again begins earlier but is relieved much sooner than for the temporal area.

evidence that indicates the participation of visual cortex. Computer simulations of rhythmic activity in cortical folds, as comprise primary visual cortex, reveal that the average power pattern across the scalp is characeristic of anatomical features of the cortical geometry (Kaufman et al. 1991b).

Moreover, suppression of spontaneous alpha rhythms in the occipital and parietal areas is found to be specific to the task (Kaufman et al. 1989). Figure 10 shows that when a subject seeks a rhyme for a word displayed on a screen, suppression over the visual area begins immediately and is sustained for more than $500 \mathrm{~ms}$. This reflects processing of the visual information in the occipital region. By comparison, after a delay of about $100 \mathrm{~ms}$ suppression begins over the temporal area and is sustained for more than $500 \mathrm{~ms}$ as the subject seeks a rhyme. The interplay between the two areas is quite apparent.

As a final example of advantages gained by measuring the neuromagentic field, we cite a recent study that is the first full use of the Sternberg paradigm to study the relation between short-term memory scanning and the spontaneous activity of the brain. The results support the hypothesis that increases in reaction time with memory set size are reflected in the duration of local changes in the level of spontaneous activity of cerebral cortex. In this study the subject was presented by earphones a series of 1,3 or 5 tones as a memory set. After a few seconds a probe tone was presented and the subject 


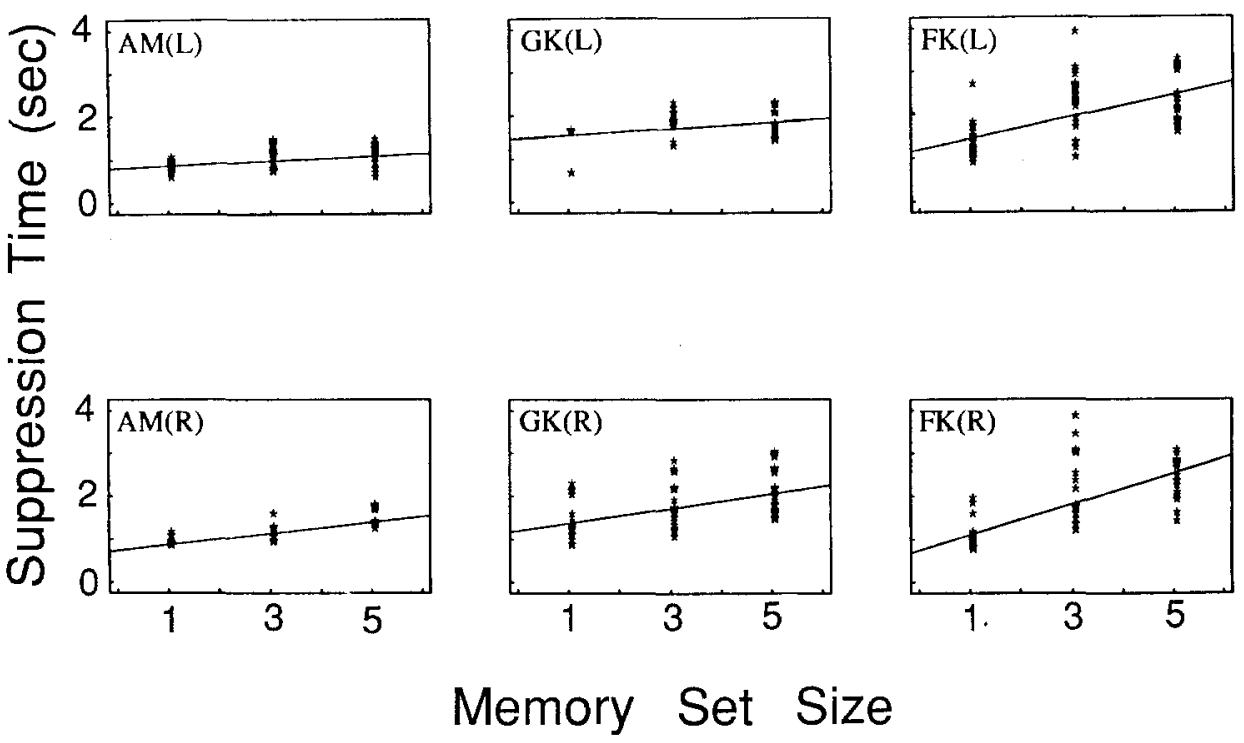

Figure 11. Duration of suppression of alpha rhythm over the left temporal area $(L)$ and right temporal area $(R)$ for each of 3 subjects after hearing a probe tone. The subject is instructed to determine whether it matches a tone previously heard as part of a memory set. Left hemisphere suppression duration is significantly correlated with memory set size for all 3 subjects' right hemisphere data, but for only one subject (FK) in the case of the left hemisphere.

pressed one reaction time button if it was judged to be a member of the memory set and another if it was not. Figure 11 shows that suppression time increases with the number of tones in the memory set (Kaufman et al. 1991), as is true for the reaction time in the classic Sternberg studies (1966). This is conventionally interpreted as reflecting the serial processing of all tones in the memory set, regardless of whether the probe occurs early in the series. Thus the duration of suppression provides a meaningful gauge of the time when a person is accessing memory.

These studies relating suppression duration to memory set size also revealed a strong correlation between the spatial pattern of suppression and the spatial distribution of $\mathrm{N} 100 \mathrm{~m}$ strength in response to presentation of the individual tones. This provides strong evidence that the alpha rhythm we observe originates in auditory cortex.

\section{Overview}

Where MSI techniques have suffered in the past, is the need to carry out sequential measurements because of the lack of a sufficient number of sensors to cover the entire scalp at one time. Determining a field pattern requires a minimum of 30 or so measurements, and often 50 or more need to be obtained for accurate results. These methods are not cost effective for a clinical setting. Moreover, such long measurement sessions may well lead to variability in responses as the state of the subject varies. Fortunate- ly, this limitation is being overcome by the advent of commercial systems that provide arrays of 37 or more sensors so that the entire field pattern from a given source can be obtained at one positioning. It is reasonable to expect that systems with more than 128 sensors will become available in the next decade to provide simultaneous measurements with proper sampling over nearly the entire head. The virtue of MEG recording being contactless means that the procedure of obtaining data can be efficient. Within five minutes or so of a subject's arrival the measurements can begin. Counterbalancing the obvious advantage in efficiency and effectiveness of large sensor arrays is the likelyhood that MSI systems will be expensive, just as for complementary imaging systems, such as positron emission tomography (PET), that provide other measures of local brain function.

MEG has a major advantage over the EEG in the fact that it provides a measurement of the field at one location in space. There is no need for a "reference". The EEG is based on the measurement of a voltage, which necessarily entails a differential measurement of the potential at two separated locations. Eliminating the effect of the reference can be done with the EEG, but it means computing the differences between two voltages, conventionally carried out by computing an approximation to the Laplacian for the data recorded across the scalp. Thus the reference is eliminated, but signals from different sources may be superimposed. Moreover, signals in the area of the scalp at the boundary of the electrode array cannot be characterized by this means, so that complete 
coverage is sacrificed. The closeness in spacing of electrodes in an array determines the accuracy with which the Laplacian can be approximated, and correspondingly the extent to which signals of different neuronal sources may be distinguished. The trade-off is that signal strength diminishes as electrodes are brought closer together. Certainly, EEG equipment is much less expensive than MEG systems, but considerable time is required to prepare the subject before, and to clean up after a study. These lengthy procedures are simply not needed for MEG recordings.

We have not discussed another advantage of MEG measurements: the bandwidth extends downward to dc. By moving a subject toward a sensor, the change in $\mathrm{dc}$ output indicates the dc field at the final position. Once in place, slow shifts in dc levels can be recorded continuously. This has advantages when characterizing the early stages of maigrane, where it is believed that spreading cortical depression may play a role (Barkley et al. 1990).

The illustrations provided in this article demonstrate how MSI can be used effectively in brain research. In the future, we expect to achieve an accurate topographic representation of cortical activity, as distinct from the topography of the MEG and EEG at the scalp. These external phenomena except in simple cases are not related to the underlying activity in any straightforward manner.

\section{References}

Bak, C., Kofoed, B., Lebech, J., Særmark, K. and Elberling, C. Auditory evoked magnetic fields from the human brain. Source locatization in a single-dipole approximation. Phys. Lett., 1981, 82A: 57-60.

Barkley, G.L., Tepley, N., Nagel-Leiby, S., Moran, J.E., Simkins, R.T. and Welch, K.M.A. Magnetoencephalographic Studies of Migraine. Headache, 1990, 30: 428-434.

Baumann, S.B., Rogers, R.L., Papanicolaou A.C. and Saydjari, C.L. Intersession replicability of dipole parameters from three components of the auditory evoked agnetic field. Brain Topography, 1990, 3: 311-319.

Brenner, D., Lipton, J., Kaufman, L. and Williamson, S.J. Somatically evoked magnetic fields of the human brain. Science, 1978, 199: 81-83.

Cohen, D., Cuffin, B.N., Yunokuchi, K., Maniewski, R., Purcell, C., Cosgrove, G.R., Ives, J., Kennedy, J.G. and Schomer, D.L. $M E G$ versus EEG localization test using implanted sources in the human brain. Ann. Neurol., 1990, 28; 811-817.

Cuffin, B.N. and Cohen, D. Magnetic fields of a dipole in special volume conductor shapes. IEEE Trans. Biomed. Eng., 1977, BME-24: 372-381.

Erné, S.N., Narici, L., Pizzella, V. and Romani, G.L. The positioning problem in biomagnetic measurements: A solution for arrays of superconducting sensors. IEEE Trans. Magn., 1987, MAG-23: 1319-1322.
Grynszpan, F. and Geselowitz, D.B. Model studies of the magnetocardiogram. Biophys. J., 1973, 13: 911-925.

Hämäläinen, M.S. and Sarvas, J. Realistic conductivity geometry model of the human head for interpretation of neuromagnetic data. IEEE Trans. Biomed. Eng., 1989, 36: 165-171.

Hari, R., Kaila, K., Katila, T., Tuomisto, T. and Varpula, T. Interstimulus interval dependence of the auditory vertex response and its magnetic counterpart: Implications for their neural generation. Electroenceph. Clin. Neurophysiol., 1982, 54: 561-569.

Hari, R., Aittoniemi, K., Järvinen, M.-L., Katila, T. and Varpula, T. Auditory evoked transient and sustained magnetic fields of the human brain: Localizaton of neural generators. Exp. Brain Res., 1980, 40:237-240.

Hari, R. and Ilmoniemi, R.J. Cerebral magnetic fields. CRC Critical Rev. in Biomed. Eng., 1986, 14: 93-126.

Hari R. and Lounasmaa, O.V. Recording and interpretation of cerebral magnetic fields. Science, 1989, 244: 432-436.

Hoke, M. SQUID-based measuring techniques - a challenge for the functional diagnostics in medicine. In: B. Kramer, (Ed.), The Art of Measurement: Metrology in Fundamental and Applied Physics, VCH Verlagsgesellschaft $\mathrm{mbH}$, Weinheim, 1988, 287-335.

Karron, D., Lï, Z.-L. and Williamson, S.J. submitted for publication, 1991.

Kaufman, L. and Price, R. The detection of cortical spike activity at the human scalp. IEEE Trans. Biomed. Eng., 1967, BME14: 84-90.

Kaufman, L. and Locker, Y. Sensory modulation of the EEG. Proc. Am. Psychology Assoc., 75th Meeting, 1970.

Kaufman, L., Okada, Y., Brenner D. and Williamson, S.J. On the relation between somatic evoked potentials and fields. Intern. J. Neuroscience, 1981, 15: 223-239.

Kaufman, L., Glanzer, M., Cycowicz, Y.M., and Williamson, S.J. Visualizing and rhyming cause differences in alpha suppression. In S.J. Williamson, M. Hoke, G. Stroink, and M. Kotani, editors, Advances in Biomagnetism, Plenum, New York, 1989, 241-244.

Kaufman, L., Schwartz, B., Salustri, C. and Williamson, S.J. Modulation of spontaneous brain activity during mental imagery. J. Cognitive Neuroscience, 1990, 2: 124-132.

Kaufman, L., Curtis, S., Wang, J.Z. and Williamson, S.J. Changes in cortical activity when subjects scan memory for tones. Electroenceph. Clin. Neurophysiol., in press, 1991.

Kaufman, L, Kaufman, J. and Wang, J.Z. On cortical folds and neuromagnetic fields. Electroenceph. Clin. Neurophysiol., 1991b, 79: 211-226.

Lü, Z.-L., Williamson, S.J. and Kaufman, L. Human auditory primary and association cortex have differing lifetimes for activation traces. Submitted 1991.

Lü, Z.-L. and Williamson, S.J. Spatial extent of coherent sensory-evoked cortical activity. Exp. Brain Res., 1991, 84: 411416.

Lütkenhöner, B., Pantev, C. and Hoke, M. Comparison between different methods to approximate an area of the human head by a sphere. In: F. Grandoli, M. Hoke, and G.L. Romani, (Eds.), Auditory Evoked Magnetic Fields and Electric Potentials, Karger, Basel, 1990, 103-118.

Näätänen R. and Picton, T. The N1 wave of the human electric 
and magnetic response to sound. Psychophysiology, 1987, 24: $375-425$.

Okada, Y.C., Williamson S.J. and Kaufman, L. Magnetic field of the human sensorimotor cortex. Intern. J. Neuroscience, 1982, 17: 33-38.

Okada, Y.C., Tanenbaum, R., Williamson, S.J. and Kaufman, L. Somatotopic Organization of the Human Somatosensory Cortex Revealed by Neuromagnetic Measurements. Exp. Brain Res., 1984, 56: 197-205.

Pandya D.N. and Seltzer, B. Association areas of the cerebral cortex. TINS, 1982, 386-390.

Pantev, C., Hoke, M., Lehnertz, K., Lütkenhöner, B., Anogianakis G. and Wittkowski, W. Tonotopic organization of the human auditory cortex revealed by transient auditory evoked magnetic fields. Electroenceph. Clin. Neurophysiol., 1988, 69: 160-170.

Pfurtscheller, G. and Aranibar, A. Event-related cortical desynchronization detected by power measurments of scalp EEG. Electroeneceph. clin. Neurophysiol., 1977, 42: 817-826.

Pfurtscheller, G., Steffan, J. and Maresch, H. ERD mapping and functional topography: Temporal and spatial aspects. In $G$. Pfurtscheller and F.H. Lopes da Silva, editors, Functional Brain Imaging, Hans Huber, Toronto, 1988, 117-130.

Pfurtscheller, G. Mapping of event related desynchronization and type of derivation. Electroenceph. Clin. Neurophysiol., 1988b, 70: 190-193.

Romani, G.L., Williamson S.J. and Kaufman, L. Tonotopic organization of the human auditory cortex. Science, 1982, 216: 1339-1340.

Romani, G.L., Williamson, S.J., Kaufman, L. and Brenner, D. Characterization of the human auditory cortex by the neuromagnetic method. Exp. Brain Res., 1982, 47: 381-393.

Rose, D.F., Ducla-Soares E. and Sato, S. Improved accuracy of MEG localization in the temporal region with inclusion of volume current effects. Brain Topography, 1989, 1: 175-181.

Scherg, M. Fundamentals of dipole source analysis. In: F. Grandori, M. Hoke, and G.L. Romani, (Eds.), Auditory Evoked
Magnetic Fields and Electric Potentials, Karger, Munich, 1990, 40-69.

Sternberg, S. High-speed scanning in human memory. Science, 1966, 153: 652-654.

Vaughan, H.G. Jr. and Ritter, W. The sources of auditory evoked responses recorded from the human scalp. Electroenceph. Clin. Neurophysiol., 1970, 28: 360-367.

Vaughan, H.G. Jr., Ritter, W. and Simson, R. Topographic analysis of auditory event-related potentials. In: H.H. Kornhuber and L. Deecke, (Eds.), Motivation, Motor and Sensory Processes of the Brain, Elsevier, Amsterdam, 1980, 279-285.

Weinberg, H., Brickett, P., Coolsma, F., and Baff, M. Magnetic localisation of intracranial dipoles: Simulation with a physical model. Electroenceph. Clin. Neurophysiol., 1986, 64: 159-170.

Williamson, S.J. and Kaufman, L. Analysis of neuromagnetic signals. In: A.S. Gevins and A. Rémond, (Eds.) Methods of Analysis of Brain Electrical and Magnetic Signals, Elsevier, Amsterdam, 1987, Chapter 14, 405-448.

Williamson, S.J. and Kaufman, L. Theory of neuroelectric and neuromagnetic fields. In F. Grandori, H. Hoke, and G.L. Romani, editors, Auditory Electric and Magnetic Fields, Karger, Basel, 1989, 1-39.

Williamson, S.J. and Kaufman, L. Advances in neuromagnetic instrumentation and studies of spontaneous brain activity. Brain Topography, 1989b, 2: 129-139.

Wolpaw, J.R. and Penry, J.K. A temporal component of the auditory evoked response. Electroenceph. Clin. Neurophysiol., 1975, 39: 609-620.

Wood, C.C. Application of dipole localization methods to source identification of human evoked potentials. Ann. N.Y. Acad. Sci., 1982, 388: 139-155.

Yamamoto, T., Williamson, S.J., Kaufman, L., Nicholson, C. and Llinás, $\mathrm{R}$. Magnetic localization of neuronal activity in the human brain. Proc. Natl. Acad. Sci. USA, 1988, 85: 87328736 . 